\title{
Utilitarianism and Discrimination*
}

\author{
Alon $\mathrm{Harel}^{\dagger}$ and Uzi Segal ${ }^{\ddagger}$
}

February 19, 2012

\begin{abstract}
Since Becker (3), a common argument against asymmetric norms that promote minority rights over those of the majority is that such policies reduce total welfare. While this may be the case, we show that there are simple environments where aggregate sum of individual utilities is actually maximized under asymmetric norms that favor minorities. We thus maintain that without information regarding individual utilities one cannot reject or promote segregation-related policies based on utilitarian arguments.
\end{abstract}

Keywords: Utilitarianism, discrimination, segregation, minority and majority rights.

\footnotetext{
*We thank Aaron Fix and Nisha Ye, and especially Inácio Guerberoff for research assistance. We thank Eddie Dekel, Larry Epstein, Johannes Hörner, Ben Polak, Larry Samuelson, and Joel Sobel for their comments.

${ }^{\dagger}$ The Hebrew University School of Law, Jerusalem, Israel (msalon@huji.ac.il) and Boston University Law Faculty.

${ }^{\ddagger}$ Dept. of Economics, Boston College, Chestnut Hill MA 02467, USA (segalu@bc. edu).
} 


\section{Introduction}

We discuss in this paper norms that facilitate or prohibit the establishment of minority-only or majority-only institutions, neighborhoods, or associations. Traditionally, asymmetric norms favoring minorities are justified on justicebased grounds, for example, by claiming that some preferences (e.g., the exclusion of blacks) ought not to be respected because they are immoral and unjust (see e.g. Harsanyi (12, p. 56) or Goodin (10)). Moreover, there are cases in which individuals have second order preferences, i.e., preferences over what preferences they want to have (see Frankfurt (9)). Thus, even without excluding preferences simply because of their pernicious content, it is possible to justify the exclusion of racist preferences to the extent that individuals wish they did not have such preferences. Another suggestion was raised by Ronald Dworkin (6, pp. 234-238). In his view preferences ought to be divided into external and internal, where external preferences are about the extent to which the preferences of other people ought to be satisfied while internal preferences do not relate to other people's desires. A fourth approach concerning segregation is based on the view that racist preferences are manipulable and may be transformed by regulating segregation.

Following Becker (3), all arguments in favor of asymmetric norms seem to suffer from one major drawback - they are inefficient in the sense that they do not maximize social utility (see for example in McAdams' (14, p. 93) criticism of this approach). Summarizing Becker's views, Posner (15) writes:

For Becker discrimination by whites against blacks is the result of an aversion that whites have to associating with blacks. This aversion makes it more costly for whites to transact with blacks than with other whites. Becker likens this additional transaction cost to transportation costs in international trade. The higher those transportation costs are, the less international trade there will be.

This paper shows that this is not necessarily the case and that asymmetric norms supporting minorities may actually increase, rather than reduce, utilitarian social welfare. We do not claim that this is always the case, but we show that under certain reasonable assumptions asymmetric norms favoring the minority would maximize aggregate sum of individual utilities. This is true even when everyone has the same utility function according to which 
individuals prefer residing near their own type rather than next to the other group.

If, as is usually the case, individual preferences are unobservable, then we cannot claim that utilitarian considerations support the establishment of black colleges or Hassidic-only neighborhoods while, at the same time, oppose exclusion of blacks or Jews from white or Christian neighborhoods. The argument of the paper is that the opposite too is false: in general, utilitarian considerations do not support the establishment of white colleges or the exclusion of of Jews from Christian neighborhoods.

To simplify the discussion we analyze a stylized problem, where people of two types (language, religion, nationality) share a country. The country is divided into up to three (disjoint, but not necessarily non-empty) parts: Two are used exclusively by members of the majority and the minority groups, while the third is shared by both types. The only difference between members of society is the majority/minority division. In particular, they all have the same preferences with respect to themselves and their respective groups in the sense that ceteris paribus they prefer to live with members of their own group but they would also like to be able to reside in as large part of the country as possible. Moreover, the larger the percentage of individuals of one's group residing in his neighborhood, the higher is the well-being of each such individual. Thus, under our view, the utility of each person is increasing with the size of the part of the country that is kept for the exclusive use of his type, with the size of the mixed area, and with his type's proportion in that region.

The paper is organized as following: Section 2 presents the basic structure of social policies. Individual preferences are discussed in Section 3 and in Appendix 1. Social welfare of policies is calculated in Section 4 and the applications of these calculations to some specific utility functions are presented in Section 5

\section{Preliminaries}

Society is composed of two groups, majority $(j)$ and minority $(n)$, with proportions $\alpha>\frac{1}{2}$ and $1-\alpha$, respectively. Society needs to allocate the use of a certain source (land, schools, etc.) between the two groups. A social policy is a pair $(\beta, \gamma)$, meaning that the majority can use $\beta \geqslant \alpha$ part of the good and the minority can use $\gamma \geqslant 1-\alpha$ part of it. For example, when $\alpha=0.8$, a 
possible social policy is to allow the majority to reside in 0.9 of the country and to allow the minority to reside in 0.4 of it. Such a policy divides the country into three parts: $0.6(=1-0.4)$ will be used exclusively by the majority, ${ }^{1} 0.1(=1-0.9)$ exclusively by the minority, and the remaining 0.3 will be inhabited by both. Of these, $0.2(=0.8-0.6)$ are members of the majority group and $0.1(=0.2-0.1)$ of the minority, hence in the $30 \%$ of the land devoted to mixed use, the proportions of the majority and the minority are two thirds and one third, respectively.

In general, we will get the following allocation. Denote by $s_{j}$ and $s_{n}$ the relative sizes of the parts of the land where only the majority and minority people can live, respectively, and denote by $t$ the size of the mixed area. Then

- Since the minority can reside only in $\gamma$ part of the country, the remaining $1-\gamma$ will have to be used solely by members of the majority. Hence $s_{j}=1-\gamma \leqslant \alpha$. The rest of the majority, that is, $\alpha-s_{j}=\alpha+\gamma-1$, live in the mixed area.

- $s_{n}=1-\beta \leqslant 1-\alpha$. The rest of the minority, $\beta-\alpha$, live in the mixed area.

- $t=1-s_{j}-s_{n}=\beta+\gamma-1$

Let $\pi_{j}$ and $\pi_{n}$ be the proportions of the two types in the mixed area. Then

$$
\pi_{j}=\frac{\alpha+\gamma-1}{\beta+\gamma-1}, \quad \pi_{n}=\frac{\beta-\alpha}{\beta+\gamma-1}
$$

and $\pi_{j}+\pi_{n}=1$. Finally, observe that

$$
s_{j}+t \pi_{j}=\alpha \text { and } s_{n}+t \pi_{n}=1-\alpha
$$

\section{Individual Preferences}

Individuals would like to reside with people like them, yet they would like to be able to live in as large part of the country as possible. ${ }^{2}$ Formally, they

\footnotetext{
${ }^{1}$ We assume throughout the same population density everywhere. In other words, $60 \%$ of the population will reside in the 0.6 part of land that is allocated for the exclusive use of the majority. Hence three quarters of the majority will live in majority-only areas.

${ }^{2}$ We use terminology that applies to housing and country division, but as stated before, college or association formations can be likewise analyzed. In such cases individuals will
} 
derive satisfaction from three variables: $s$, the size of the area that is assigned exclusively for their group; $t$, the size of the mixed area, and the proportion $\pi$ of their type in this mixed area. Of course, for a given group in a given society, it is impossible to increase all three variables and there is a trade-off between the possible triples each group will face. For example, increasing the size of the mixed area (a change favored by the majority) at the cost of reducing the exclusive area of the minority will reduce the proportion of the majority in the mixed area (a change they dislike). The preferences we discuss are abstract and relate to hypothetical states, existing or not. These preferences may or may not be related to actual benefits from interaction with other individuals of the same type: They may depend on cultural affiliation with people of the same type or from true benefits a person receives from interacting with his type (as in Athey, Avery, and Zemsky (2)).

We assume that everyone has the same preferences over triples $(s, t, \pi)$. That is, the asymmetric results we obtain do not follow from asymmetric preferences. As the aim of the present analysis is to show the possibility that the optimal policy restrains the majority but not the minority (that is, $\beta<1$ and $\gamma=1$ ), the results will be more convincing if individual preferences are as simple as possible. We will therefore analyze the case where these preferences can be represented by the function $u$, given by

$$
u(s, t, \pi)=s+\operatorname{tg}(\pi)
$$

where $g$ is strictly increasing, $g(0)=0$ and $g(1)=1$. As we show in the Appendix, these preferences can be derived from some simple axioms. We assume that this is the utility of a person independently of the location of his residence - in the segregated or in the mixed area. In other words, our analysis is ex ante, before anyone knows where exactly he'll live, rather than ex post (on the basis of the utility gained from living in either a mixed or a homogenous area). Under our model, people gain utility not from living in a specific area (which is either mixed or homogenous) but from living in a jurisdiction which has certain rules governing the allocation of rights to different groups.

We maintain that the ex ante perspective is indeed better than the ex post one as we assume that people derive satisfaction not just from the density of their type in their immediate neighborhood, but also from the size of the set

prefer to study or associate with people like them, while also preferring to have as much choice as possible. 
of options available to them. Therefore the appropriate attitude to options available to a person is when they are viewed ex ante, before he knows where he is going to settle.

There is another reason why we believe that the correct perspective is ex ante and not ex post. Recall that our analysis applies to many spheres of life, like neighborhoods, schools, social clubs, restaurants, offices, etc. Given the broad scope of our analysis the rules governing the allocation of rights of different groups become primary in determining the utility of individuals during the course of their lives. Suppose that one's utility is the sum of the utilities derived from the composition of each one of the institutions to which a person belongs as well as the expectations concerning the institutions to which one will belong in the future. Any rule concerning segregation (a rule that prohibits segregation, an asymmetrical rule favoring minorities or a symmetrical rule) will have applications with respect to all spheres of life. Furthermore, it will have applications that extend to the expectations of individuals concerning the future as people may join new clubs and leave old ones. The overall utility is therefore a function of the ex ante perspective (based on the rules governing the permissibility of segregation) rather than a function of the ex post perspective.

Following the analysis of the previous section, the policy $(\beta, \gamma)$ leads members of the majority to the utility

$$
\begin{aligned}
u\left(s_{j}, t, \pi_{j}\right)= & u\left(1-\gamma, \beta+\gamma-1, \frac{\alpha+\gamma-1}{\beta+\gamma-1}\right)= \\
& 1-\gamma+(\beta+\gamma-1) g\left(\frac{\alpha+\gamma-1}{\beta+\gamma-1}\right)
\end{aligned}
$$

while the utility of members of the minority will be

$$
\begin{aligned}
u\left(s_{n}, t, \pi_{n}\right)= & u\left(1-\beta, \beta+\gamma-1, \frac{\alpha+\gamma-1}{\beta+\gamma-1}\right)= \\
& 1-\beta+(\beta+\gamma-1) g\left(\frac{\beta-\alpha}{\beta+\gamma-1}\right)
\end{aligned}
$$

Observe that if $g(\pi)=\pi$ for all $\pi$, then individuals don't care how the country is divided, as the utility of the majority is $\alpha$ and the utility of the minority is $1-\alpha$, regardless of the actual division.

Remark: The preferences discussed above are different from the expected ex post utilities restricted to the various areas. Formally, one can suggest a 
model where the utility of those who live in the segregated area equals the size of that area, and the utility of those who live in the mixed area is the size of the area multiplied by $g(\pi)$ where $\pi$ is the proportion of their type in this area. Finally, the ex ante utility will be the expected utility from the two areas, where the weights of the two utilities are proportional to the division of the type between them. According to this proposal, the policy $(\beta, \gamma)$, leading to the division $1-\gamma$ for the majority only, $1-\beta$ for the minority only, and $\beta+\gamma-1$ mixed area, yields $1-\gamma$ members of the majority utility $1-\gamma$ and it yields the remaining $\alpha+\gamma-1$ members of the majority utility $(\beta+\gamma-1) g\left(\frac{\alpha+\gamma-1}{\beta+\gamma-1}\right)$. The expected value of these utilities is

$$
\frac{1-\gamma}{\alpha} \times(1-\gamma)+\frac{\alpha+\gamma-1}{\alpha} \times(\beta+\gamma-1) g\left(\frac{\alpha+\gamma-1}{\beta+\gamma-1}\right)
$$

When $g$ is linear, this expression reduces to $\frac{1}{\alpha}\left[(1-\gamma)^{2}+(\alpha+\gamma-1)^{2}\right]$, hence it is sensitive to changes in $\gamma$. As we saw above with respect to our model, when $g$ is linear, individual utilities are not sensitive to social policies.

\section{Social Welfare}

If everyone prefers full segregation or if everyone prefers no segregated areas, the social optimum will agree with these preferences. This is the case when $g$ is either convex or concave.

If $g$ is convex (in fact, even if for every $0<\pi<1, g(\pi) \leqslant \pi$ ), then

$$
u(s, t, \pi)=s+t g(\pi) \leqslant s+t \pi=u(\alpha, 0,0)
$$

where the last equation-sign follows by eq. (1). In this case, everyone in society (weakly) prefers full segregation $(t=0)$, and therefore any social preferences satisfying unanimity will rank this policy best. On the other hand, if $g$ is concave, then having no exclusive areas is optimal (see proposition 2 below). We now turn to the construction of individual and social preferences that will lead society to restrict only the majority, but not the minority. We assume that society has preferences over policies, that is, over pairs $(\beta, \gamma)$. Following Harsanyi (11) (but assuming same weights to all), we assume that these preferences can be represented by the sum of individual utilities. This form of utilitarianism seems to us to serve our purpose everyone counts, and no one has higher value than others. 
As the proportion of the majority is $\alpha$, it follows by eqs. (2) and (3) that society is looking to maximize, with respect to $\beta$ and $\gamma$, the function $W$ given by

$$
\begin{aligned}
& W(\beta, \gamma)=\alpha u\left(s_{j}, t, \pi_{j}\right)+(1-\alpha) u\left(s_{n}, t, \pi_{n}\right)= \\
& \alpha\left[1-\gamma+(\beta+\gamma-1) g\left(\frac{\alpha+\gamma-1}{\beta+\gamma-1}\right)\right]+ \\
& \quad(1-\alpha)\left[1-\beta+(\beta+\gamma-1) g\left(\frac{\beta-\alpha}{\beta+\gamma-1}\right)\right]= \\
& 1-\alpha \gamma-(1-\alpha) \beta+ \\
& \quad(\beta+\gamma-1)\left[\alpha g\left(\frac{\alpha+\gamma-1}{\beta+\gamma-1}\right)+(1-\alpha) g\left(\frac{\beta-\alpha}{\beta+\gamma-1}\right)\right]
\end{aligned}
$$

The next proposition shows that if the optimal policy is not full separation, then at least one of the two groups should not be restricted at all.

Proposition 1 The maximum of the above function $W$ of eq. (4) is at one (or more) of the following points:

1. $\beta=\alpha, \gamma=1-\alpha$,

2. $\beta=1$,

3. $\gamma=1$.

Proof: Obviously, if $\beta=\alpha$ [alt. $\gamma=1-\alpha$ ] then effectively $\gamma=1-\alpha$ [alt. $\beta=\alpha$ ]. The claim thus rules out the possibility that $\alpha<\beta<1$ and $1-\alpha<\gamma<1$. Suppose that social welfare is maximized at $\left(\beta^{*}, \gamma^{*}\right) \in$ $(\alpha, 1) \times(1-\alpha, 1)$. Define $\kappa=\left(\gamma^{*}+\alpha-1\right) /\left(\beta^{*}-\alpha\right)$ and consider the function

$$
\begin{aligned}
F(\xi)= & W(\alpha+\xi, 1-\alpha+\kappa \xi)= \\
& 1-2 \alpha(1-\alpha)-\alpha \kappa \xi-(1-\alpha) \xi+ \\
& \xi(1+\kappa)\left[\alpha g\left(\frac{\kappa}{1+\kappa}\right)+(1-\alpha) g\left(\frac{1}{1+\kappa}\right)\right]
\end{aligned}
$$

For $\xi=\beta^{*}-\alpha, F(\xi)=W\left(\beta^{*}, \gamma^{*}\right)$. The function $F$ is linear in $\xi$, hence is maximized either at $\xi=0$ (case 1 of the claim) or when either $\alpha+\xi=1$ (case 2 ) or $1-\alpha+\kappa \xi=1$ (case 3 ).

The following claim follows from the last proposition: 
Proposition 2 If $g$ is concave then $(\beta, \gamma)=(1,1)$ is optimal. In other words, having no segregated areas is not inferior to any other policy.

Proof: By the concavity of $g$,

$$
W(1,1)=\alpha g(\alpha)+(1-\alpha) g(1-\alpha) \geqslant \alpha^{2}+(1-\alpha)^{2}=W(\alpha, 1-\alpha)
$$

Hence we should check cases 2 and 3 in proposition 1 . Suppose $\beta=1$ is optimal and compare it with the case $(\beta, \gamma)=(1,1)$. Given policy $(1, \gamma)$, $1-\gamma$ members of the majority live in an exclusive area. The remaining $\alpha+\gamma-1$ members of the majority together with all $1-\alpha$ members of the minority live in the mixed area. The size of the mixed area is $\gamma$, and the corresponding proportions there are $\frac{\alpha+\gamma-1}{\gamma}$ and $\frac{1-\alpha}{\gamma}$. The utility of each member of the minority under policy $(1, \gamma)$ is therefore

$$
\begin{aligned}
\gamma g\left(\frac{1-\alpha}{\gamma}\right)= & \gamma g\left(\frac{1-\alpha}{\gamma}\right)+(1-\gamma) g(0) \leqslant \\
& g\left(\gamma \times \frac{1-\alpha}{\gamma}+(1-\gamma) \times 0\right)=g(1-\alpha)
\end{aligned}
$$

where the inequality follows by the concavity of $g$. Likewise, the utility of each member of the majority is

$$
\begin{aligned}
1-\gamma+\gamma g\left(\frac{\alpha+\gamma-1}{\gamma}\right)= & (1-\gamma) g(1)+\gamma g\left(\frac{\alpha+\gamma-1}{\gamma}\right) \leqslant \\
& g\left((1-\gamma) \times 1+\gamma \times \frac{\alpha+\gamma-1}{\gamma}\right)=g(\alpha)
\end{aligned}
$$

Hence $W(1,1) \geqslant W(1, \gamma)$. Similarly, $W(1,1) \geqslant W(\beta, 1)$.

\section{$5 \quad$ Examples}

In this section we show that all the cases of proposition 1 may happen. In particular, we show that there are functions $g$ such that the unique optimal social policy is to set $\gamma=1$ and $\beta<1$. Such policies restrict the majority without putting any restrictions on the minority.

According to proposition 1 we have to check for maximum points of $W(\beta, 1)$ with respect to $\beta$ and of $W(1, \gamma)$ with respect to $\gamma$, and compare 
them with $W(\alpha, 1-\alpha)$. Setting $\gamma=1$ in eq. (4) we obtain that society should maximize, as a function of $\beta$,

$$
(1-\alpha)(1-\beta)+\beta\left[\alpha g\left(\frac{\alpha}{\beta}\right)+(1-\alpha) g\left(1-\frac{\alpha}{\beta}\right)\right]
$$

First and second order conditions for maximization with respect to $\beta$ are

$$
\begin{aligned}
& -(1-\alpha)+\alpha g\left(\frac{\alpha}{\beta}\right)+(1-\alpha) g\left(1-\frac{\alpha}{\beta}\right) \\
& -\frac{\alpha^{2}}{\beta} g^{\prime}\left(\frac{\alpha}{\beta}\right)+\frac{\alpha(1-\alpha)}{\beta} g^{\prime}\left(1-\frac{\alpha}{\beta}\right)=0 \\
& \alpha g^{\prime \prime}\left(\frac{\alpha}{\beta}\right)+(1-\alpha) g^{\prime \prime}\left(1-\frac{\alpha}{\beta}\right)<0
\end{aligned}
$$

Likewise, setting $\beta=1$ in eq. (4) we obtain that society should maximize, as a function of $\gamma$,

$$
\alpha(1-\gamma)+\gamma\left[\alpha g\left(1-\frac{1-\alpha}{\gamma}\right)+(1-\alpha) g\left(\frac{1-\alpha}{\gamma}\right)\right]
$$

First and second order conditions for maximization with respect to $\gamma$ are

$$
\begin{aligned}
& -\alpha+\alpha g\left(1-\frac{1-\alpha}{\gamma}\right)+(1-\alpha) g\left(\frac{1-\alpha}{\gamma}\right)+ \\
& \frac{\alpha(1-\alpha)}{\gamma} g^{\prime}\left(1-\frac{1-\alpha}{\gamma}\right)-\frac{(1-\alpha)^{2}}{\gamma} g^{\prime}\left(\frac{1-\alpha}{\gamma}\right)=0 \\
& \alpha g^{\prime \prime}\left(1-\frac{1-\alpha}{\gamma}\right)+(1-\alpha) g^{\prime \prime}\left(\frac{1-\alpha}{\gamma}\right)<0
\end{aligned}
$$

Consider first the case where $g(1-\pi)=1-g(\pi)$. We focus attention on two special cases of this form: (i) $g$ is convex on $\left[0, \frac{1}{2}\right]$ and concave on $\left[\frac{1}{2}, 1\right]$ and (ii) $g$ is concave on $\left[0, \frac{1}{2}\right]$ and convex on $\left[\frac{1}{2}, 1\right]$. Case (i) represents individuals who get most of their satisfaction when they become the majority in the mixed area and those who were the majority in the mixed area suffer the sharpest decline in utility when they become the minority there. Case (ii) represents the opposite attitude - individuals enjoy most the fact that their type "made it" into the segregated area, and suffer most from the first people of the other type to enter their domain.

If $g(1-\pi)=1-g(\pi)$, then $g^{\prime}(1-\pi)=g^{\prime}(\pi)$ and $g^{\prime \prime}(1-\pi)=-g^{\prime \prime}(\pi)$. Set $\gamma=1$ and obtain from eqs. (6) and (7)

$$
g^{\prime}\left(\frac{\alpha}{\beta}\right)=\frac{g(\alpha / \beta)}{\alpha / \beta} \text { and } g^{\prime \prime}\left(\frac{\alpha}{\beta}\right)<0
$$


(recall that $\alpha>\frac{1}{2}$ hence $2 \alpha-1>0$ ). An inner solution is thus possible in case (i), but not in case (ii) (see Fig. 1). Observe however that the solution is indeed an inner solution only if $\beta \in(\alpha, 1)$, that is, if $\frac{\alpha}{\beta} \in(\alpha, 1)$.

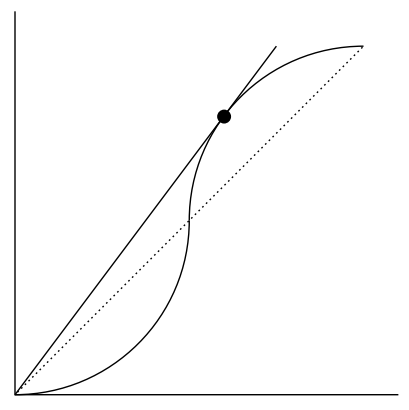

Case (i)

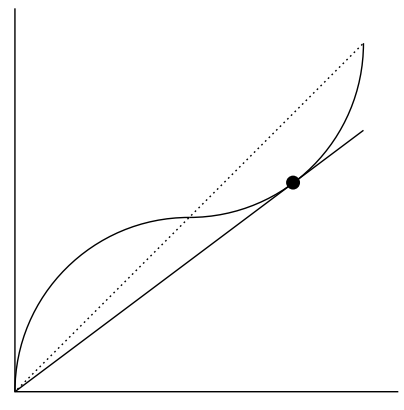

Case (ii)

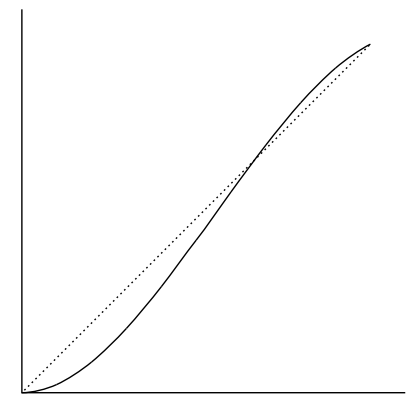

$g(\pi)=2.5 \pi^{2}-1.5 \pi^{3}$

Figure 1: The function $g$

Alternatively, set $\beta=1$ and obtain from eqs. (8) and (9)

$$
g^{\prime}\left(\frac{1-\alpha}{\gamma}\right)=\frac{g([1-\alpha] / \gamma)}{[1-\alpha] / \gamma} \text { and } g^{\prime \prime}\left(\frac{1-\alpha}{\gamma}\right)>0
$$

Here an inner solution is possible in case (ii), but not in case (i). We obtain that if full segregation is not optimal, then in case (i) it is optimal to restrict the majority but not the minority (that is, set $\gamma=1$ and $\beta<1$ ), while in case (ii) it is optimal to restrict the minority but not the majority.

The symmetry assumption $g(1-\pi)=1-g(\pi)$ seems strong, but it is more plausible in case (i) than in case (ii). If individuals care about their type being the minority or the majority in the mixed region, then we should expect $g$ to have the highest slope on both sides of $\frac{1}{2}$. In fact, We can get the optimum of $\gamma=1>\beta$ even if the crucial point is not $\frac{1}{2}$, for example, individuals may care most about whether their type consists of more or less than $\frac{2}{3}$ of the mixed region. Consider, for example, the function $g(\pi)=2.5 \pi^{2}-1.5 \pi^{3}$ (third panel in Fig. 1). This function crosses the main diagonal at $\pi=\frac{2}{3}$. For $\alpha \geqslant \frac{2}{3}$, the optimal division is $\gamma=1$ and $\beta=\left(12 \alpha^{2}-6 \alpha\right) /(9 \alpha-4)$ (see attached file for details). Observe that $\beta \geqslant \alpha$ iff $\alpha \geqslant \frac{2}{3}$.

We use the rest of the section to provide some intuition for our results. Under the assumption of diminishing marginal utility, a utilitarian society should transfer money from the rich to the poor. Our results seem to be in line with this observation - just replace "rich" with "majority" and "poor" 
with "minority." This intuition, however, is wrong. The fact that the optimal policy restricts the majority but not the minority does not necessarily mean that this is the policy members of the minority prefer most. In the above example, where $g(\pi)=2.5 \pi^{2}-1.5 \pi^{3}$, the optimal division when $80 \%$ of the population belong to the majority $(\alpha=0.8)$ is to let the minority live everywhere and to restrict the majority to $90 \%$ of the country $(\gamma=1, \beta=$ $0.9)$. The utility of each member of the minority under this policy is 0.13 , far below the utility level 0.2 obtained by them from full segregation.

We offer instead the following intuition. Consider Fig. 1. If the majority is large, then whatever policy we use most members of this group will be in areas where they'll form a large majority. In case (i), most of them will suffer only a small loss of utility whatever policy we use, because their marginal loss of utility from moving from an exclusive area to a mixed area where they are the majority is small. On the other hand, establishing some exclusive areas for the minority will add significantly to the utility of members of this group, because, once again, the shape of the utility function of the left panel indicates that their utility from being the minority is even less than their proportion in the area.

The situation in the mid-panel is of course different. Here the loss in utility to members of the majority from moving to a mixed area is significant, and moreover, the utility members of the minority will gain from moving from a mixed area to an exclusive one is less than in the left panel. This intuition explains why we get that minority-only areas are optimal when the utilities are as in the left (and right) panel, but majority-only areas are optimal when utilities are as in the center panel.

\section{Concluding Remarks}

This paper is not intended to justify the current legal system that permits the establishment of minority-only institutions, neighborhoods, or associations and it certainly does not claim that utilitarian arithmetic justifies such legislation. Nor is it our goal to compute optimal policies of land allocation. ${ }^{3}$ Yet we maintain that the automatic denial of the possibility that utilitarian considerations may justify the establishment of minority-only privileges is too hasty. Our analysis dictates caution on the part of those who use util-

\footnotetext{
${ }^{3}$ It may well be that maximal social welfare requires a division of the state into several mixed areas with different minority/majority ratios.
} 
itarianism to attack progressive legislation establishing asymmetrical norms (e.g. Posner (15)).

The paper shows that asymmetric norms that favor minorities may maximize a utilitarian social welfare function in cases individuals benefit greatly from increasing their group's presence in a neighborhood above a certain threshold, e.g., 50\%. Of course the precise threshold and the impact on one's well-being may differ from one case to another. Yet under such circumstances utilitarian considerations may dictate the establishment of an affirmative action regime in which minorities are privileged over majorities. Is this assumption realistic?

One response is that our paper does not aim to establish that the circumstances which justify asymmetrical norms hold in reality. We have no information concerning the utility functions of individuals and therefore we cannot affirm that such a system is indeed optimal. Yet, it is also evident that the more plausible the conditions, the stronger is our case. Hence it is valuable to establish that the assumptions which give rise to the justifiability of the affirmative action scheme are indeed plausible even if not necessarily true.

We believe that the threshold conditions we posit are plausible. One reason people prefer the density of their type to be high is not necessarily that they don't want to see the other type, but that they wish to meet their own type as effortlessly as possible. Once the density of their type reaches a certain level, individuals are very likely to meet and interact with their own type. Beyond this level, higher density will yield a diminishing marginal contribution to their utility. On the other hand, when their density is low, especially when it is very low, members are very unlikely to meet people of their type regardless of their efforts.

Other reasons why the threshold condition is plausible involve arguments that go beyond the extended Beckerian preferences we analyzed. For example, the presence of a certain percentage, e.g., $50 \%$ of members of one's group guarantees certain impact on the political system. If the different groups have different political tastes, it follows that it will be crucial for a group to reach a threshold (this threshold is not necessarily one half) which entitles it to a share in the local government of the mixed area. At times the desirable threshold may be sufficient to block unfriendly amendments proposed by an adverse group. Alternatively the desirable threshold is sufficient to pass laws which promote the interests of the group (at the expense of the adverse group). Consequently a small increase in the presence of the relevant 
group around the threshold may trigger a great increase in the well-being of its members.

Secondly, the presence of a certain percentage of members of the relevant group may affect the supply of goods and services. The greater their presence, the more likely there will be businesses which provide goods and services catering to their taste. Such an advantage often depends on reaching a certain threshold of presence in the relevant neighborhood. One can for instance imagine that the presence of ten thousands Hispanics (comprising of $30 \%$ of the population) may make it profitable to open a book store specializing in books in Spanish. The presence of thirty thousand Hispanics makes it profitable to open a cinema specializing in Spanish-speaking movies etc. Once again it seems that a small increase in the percentage of Hispanics around the threshold may trigger a large increase in their well-being. Therefore, although the paper shows that different utility functions may lead to different optimal allocations of rights, we believe that there are good reasons to assume that actual preferences are such that granting extra rights to minorities are optimal. Both of the last two arguments, the political and the cultural, rely on preferences for goods and services that can be supplied without interfering with the racial compositions of different neighborhoods.

Our main point however is not that utilitarian calculus implies that society should prefer minorities over majorities. Rather, we showed that the opposite is not necessarily true, that is, affirmative action policies do not

necessarily reduce social welfare but may actually maximize social sum of utilities. A natural question this paper did not attempt to answer is how should society react to situations where a minority group is trying to exclude another minority group (for example, religious groups trying to exclude homosexuals, black colleges rejecting Hispanic candidates, etc.) Utilitarian calculus may help answering such questions.

\section{Appendix: Axioms}

In this appendix we offer axioms leading to the conclusion that the utility of each person from a policy leading to $(s, t, \pi)$ is

$$
u(s, t, \pi)=s+\operatorname{tg}(\pi)
$$

where $g$ is strictly increasing, $g(0)=0$ and $g(1)=1$. 
Consider the set $A=\left\{(s, t, \pi) \in[0,1]^{3}: s+t \leqslant 1\right\}$. From an individual point of view, the vector $(s, t, \pi)$ means that the group to which the individual belongs can reside in $s+t$ part of the country, where in the $s$ part only this group resides while in the $t$ part both groups can reside, and the proportion of people of the individual's group in this $t$ area is $\pi$. Let $\succeq$ be a complete, transitive, and continuous preference relation over $A$. There is therefore a function $u$ representing these preferences. Assume further that $\succeq$ is increasingly monotonic in $s$, and for positive $t$ and $\pi$, it is increasing in these two variables as well.

When $\pi=0$, there are no representatives of the individual's type in the mixed area, so this area does not really exist. This leads to the first restriction on $\succeq$ :

A. For all $t \leqslant 1-s,(s, t, 0) \sim(s, 0,0)$.

Similarly, if $t=0$ there is no mixed area, and $\pi$ doesn't matter. That is,

B. For all $\pi,(s, 0, \pi) \sim(s, 0,0)$.

When $\pi=1$, the mixed area is not mixed but is inhabited by members of the individual's group only. Hence

C. $(s, t, 1) \sim(s+t, 0,0)$.

Next, we assume separability between the two areas. The evaluation of two possible mixed areas does not depend on the size of what is assigned to the exclusive use of the individual's group. Formally:

D. $(s, t, \pi) \succeq\left(s, t^{\prime}, d^{\prime}\right) \Longleftrightarrow\left(s^{\prime}, t, \pi\right) \succeq\left(s^{\prime}, t^{\prime}, d^{\prime}\right)$.

Define $f(t, \pi)$ by $(0, t, \pi) \sim(0, f(t, \pi), 1)$ and obtain by the last two assumptions that

$$
(s, t, \pi) \sim(s, f(t, \pi), 1) \sim(s+f(t, \pi), 0,0)
$$

Therefore $\succeq$ can be represented by

$$
u(s, t, \pi)=s+f(t, \pi)
$$

Observe that by assumption $\mathrm{C}$ above,

$$
f(t, 1)=t
$$

Finally, we assume size-homotheticity: 
E. $(s, t, \pi) \succeq\left(s^{\prime}, t^{\prime}, d^{\prime}\right)$ iff for all appropriate ${ }^{4} \lambda,(\lambda s, \lambda t, \pi) \succeq\left(\lambda s^{\prime}, \lambda t^{\prime}, d^{\prime}\right)$. In particular, $(s, t, 1) \sim\left(s, t^{\prime}, \pi\right)$ implies $(\lambda s, \lambda t, 1) \sim\left(\lambda s, \lambda t^{\prime}, \pi\right)$. By eqs. $(10)$ and (12),

$$
\begin{aligned}
& t=f(t, 1)=f\left(t^{\prime}, \pi\right) \\
& \lambda t=f(\lambda t, 1)=f\left(\lambda t^{\prime}, \pi\right)
\end{aligned}
$$

As $f$ is homogeneous of degree 1 in its first argument, it follows that $u(s, t, \pi)$ $=s+t g(\pi)$ (see eq. (11) above). Since $u(s, t, 0)=s$ it follows that $g(0)=0$, and since $f(t, 1)=t$ it follows that $g(1)=1$.

\section{References}

[1] Adler, Matthew D. and Eric A. Posner, 2000. "Implementing costbenefit analysis when preferences are distorted," Journal of Legal Studies 29:1105-1147.

[2] Athey, Susan, Christopher Avery, and Peter Zemsky, 2000. "Mentoring and diversity," American Economic Review 90:765-86.

[3] Becker, Gary S., 1971. The Economics of Discrimination, 2nd ed. University of Chicago Press, 1971.

[4] Bell, Derrick A., 1979. "Black colleges and the desegregation dilemma," Emory Law Journal 28:949-984.

[5] Dau-Schmidt, Kenneth G., 1990. "An economic analysis of the criminal law as a preference-shaping policy," Duke Law Journal 1990:1-38.

[6] Dworkin, Ronald, 1977. "Reverse discrimination," in R. Dworkin (ed.): Taking Rights Seriously. London: Duckworth.

[7] Eastland, Tery, 1997. Ending Affirmative Action: The Case for Color blind Justice. New York: Basic Books.

[8] Ely, John Hart, 1983. "Professor Dworkin's external/personal preference distinction," Duke Law Journal 1983 (5):959-986.

\footnotetext{
${ }^{4}$ That is, for all $0<\lambda \leqslant \min \left\{\frac{1}{s+t}, \frac{1}{s^{\prime}+t^{\prime}}\right\}$.
} 
[9] Frankfurt, Harry, 1971. "Freedom of the will and the concept of a person," The Journal of Philosophy 68:5-20.

[10] Goodin, Robert E., 1986. "Laundering preferences," in J. Elster and A. Hylland (eds.): Foundations of Social Choice Theory, 133-164. NY: Cambridge UP.

[11] Harsanyi, John C., 1955. "Cardinal welfare, individualistic ethics, and interpersonal comparisons of utility," Journal of Political Economy 63:309-321.

[12] Harsanyi, John C., 1982. "Morality and the theory of rational behavior," in A. Sen and Williams, eds., Utilitarianism and Beyond, 39-62.

[13] Luti, Antony N., 1999. "When a door closes, a window opens: Do today's private historically black colleges and universities run afoul of conventional equal protection analysis?" 42 Howard Law Journal 42:469-504.

[14] McAdams, Richard, 2002. "Relative preferences," 102 Yale Law Journal $1: 91$.

[15] Posner, Richard A., 1987. "The efficiency and the efficacy of Title VII," University of Pennsylvania Law Review 136:513.

[16] Regan, Donald, 1978. "Glosses on Dworkin: Rights, principles, and policies," Michigan Law Review 76:1213-1264. 


\section{The Case $g(\pi)=2.5 \pi^{2}-1.5 \pi^{3}$}

Consider ${ }^{5}$ the function $g(\pi)=2.5 \pi^{2}-1.5 \pi^{3}$. Set $\gamma=1$ and obtain from eq. (6)

$$
\beta=\frac{12 \alpha^{2}-6 \alpha}{9 \alpha-4}
$$

Observe that $\beta \geqslant \alpha$ iff $\alpha \geqslant \frac{2}{3}$. Substitute into the second order condition to obtain $-2 \alpha+1$ which is negative for all $\alpha>\frac{1}{2}$, hence $\left(\frac{12 \alpha^{2}-6 \alpha}{9 \alpha-4}, 1\right)$ is a maximum point.

Next we check for the optimal value of $\gamma$ when $\beta=1$. Similarly to the above analysis we get from eq. (8) that

$$
\gamma=\frac{30 \alpha-54 \alpha^{2}+42 \alpha^{3}-12 \alpha^{4}-6}{19 \alpha-23 \alpha^{2}+9 \alpha^{3}-5}
$$

Substitute into the second order condition to obtain

$$
\frac{(9 \alpha-5)^{4}}{432(2 \alpha-1)^{3}(1-\alpha)}
$$

which is positive for all $\alpha>\frac{1}{2}$ (and $\neq \frac{5}{9}$, where it is zero). In other words, when $\beta=1$ the optimal value of $\gamma$ is either 1 (which is covered by the previous analysis), or $1-\alpha$. We have therefore to check the value of $W(\beta, \gamma)$ at two points, $(\alpha, 1-\alpha)$ and $\left(\frac{12 \alpha^{2}-6 \alpha}{9 \alpha-4}, 1\right)$. From eq. (5) we obtain

$$
\begin{aligned}
& W\left(\frac{12 \alpha^{2}-6 \alpha}{9 \alpha-4}, 1\right)-W(\alpha, 1-\alpha)= \\
& \frac{\left(76 \alpha-171 \alpha^{2}+135 \alpha^{3}-12\right) \alpha}{72(2 \alpha-1)^{2}}
\end{aligned}
$$

The sign of this expression is the same as the sign of $76 \alpha-171 \alpha^{2}+135 \alpha^{3}-12$. This function is increasing on $\left[\frac{1}{2}, 1\right]$ and its value at $\alpha=\frac{2}{3}$ is positive. In other words, for all $\alpha>\frac{2}{3}$, the optimal policy is to set $\beta=\frac{12 \alpha^{2}-6 \alpha}{9 \alpha-4}$ and $\gamma=1$.

\footnotetext{
${ }^{5}$ This section is not intended to be part of the paper but to explain the calculations leading to the results related to the function $g(\pi)=2.5 \pi^{2}-1.5 \pi^{3}$ in section 5 .
} 